\title{
Bridging the Strategic Gap Between Biotech and Pharma Companies
}

\author{
Gudrun Tiedemann*
}

\begin{abstract}
Although the pharmaceutical world market is larger than $€ 400$ billion and shows an annual growth rate of $10 \%$, there is a lack of new innovative products. Many life sciences start-ups have been founded in the last decade and are pursuing promising new approaches to cover medical needs. However, there is a 'strategic gap' between discovery and development which is difficult to finance. In Germany with its several hundred biotech startups and medium-size pharma companies, new ways are being explored how to overcome this strategic gap and move new products to the market.
\end{abstract}

Keywords: Competence modules · German health law · German pharmaceutical industry · Pharma- and biotech companies · Strategic gap

\section{Introduction}

The pharmaceutical market with a global value of $€ 413$ billion and a $10 \%$ growth rate per year is a strongly growing market. The demand for medical care will further increase because of the aging population in the developed countries and the industrialization of Asia. Beyond that the tremendous advances in basic sciences are accelerating opportunities for new therapeutic discovery and developments. This revolution in biomedical science raises new hope for the prevention, treatment, and cure of serious illnesses.

Most of the ideas for modern drugs come from biotechnology: there is a paradigm shift in drug development from

${ }^{*}$ Correspondence: Dr. G. Tiedemann

Senior Vice President

Biotechnology / R\&D

German Pharmaceutical Industry Association

(Bundesverband der Pharmazeutischen Industrie BPI)

Robert-Koch-Platz 4

D-10115 Berlin, Germany

Tel.: +49 3027909160

E-Mail: gtiedemann@bpi.de chemical to biological drugs. Today there are more than hundred biological drugs on the market!

\section{The Challenge Today}

The challenge today is to ensure that basic discoveries turn quickly into new and better medical treatments. But the path from idea to innovation in the pharmaceutical business is a complex process with high risks. One has to progress successfully along a multidimensional path from discovery to commercial marketing. And this path is costly and time consuming. The total investment required for one drug launch is up to $\$ 1$ billion and the development time is on average 11 years! There are strong regulations from the health authorities assuring quality, safety and efficacy on the way. And at the end there is no real market: the availability to patients is given by law whereby pricing and scope of reimbursement differ widely in the European countries.

\section{The Players}

The players on this development path are manifold. Scientists are one of them because ideas come from basic discoveries and applied sciences. These ideas need to be patented and transferred into the economic process. Often this process begins with the foundation of biotech startup companies. In these companies know- how in drug discovery and research is abundant and they bring the ideas forward. But what they miss is experience in drug development, production, marketing and sales, which is available in the pharmaceutical industry. So the pharmaceutical industry is another important player. Today the pharma companies prefer to fill the pipelines by cooperation and partnering. They cannot have all the new technologies in-house.

The big, global pharmaceutical companies concentrate more on blockbusters with potentially high market return, whereas the hundreds of midsize pharmaceutical companies, which we have particularly in Germany, focus more on specialty pharma products. These established pharma companies are in a process of change in Germany because of health policy from the new law (Gesundheitsmodernisierungs-Gesetz, GMG). Many of these companies have substantial knowhow in drug development and are more than ever keen to develop innovations in cooperation with biotech companies or scientific institutions.

Further players on the development path are politics and authorities, who promote and protect public health, and those who are responsible for the economic environment. They are confronted with the task to create an innovation friendly framework. It is a global competition. If we cannot develop innovations speedily in Germany, others will do it!

And last but not least there are the financiers. Their part of the job is to offer ad- 
equate finance instruments. They must adapt the right means to the end. Therefore they need patience and they have to invest a lot of money.

\section{Worldwide 'Stagnation'}

Cooperation between science, biotech and pharma is more than ever a challenge. In big pharma there is today a worldwide 'stagnation'. Several important drugs are expected to lose patent protection. Despite a large increase in $R \& D$, the number of new chemical entities (NCEs) has risen only slightly and since 2000 there is a slowdown in new drug submissions to regulatory agencies worldwide. Concern is growing that many of the new basic science discoveries may not quickly yield more effective, affordable and safe drugs for patients. It is realized that the pace of drug-development work has not kept up with the rapid advances in discovery and development.

\section{The German Biotech/Pharma Location}

When we look at the German biotech/pharma location we see some hundred biotech companies. These have been founded in the mid-nineties. They are innovative and have know-how in drug discovery. But their finance- and knowhow resources for drug development are limited and up to now they have not registered a biopharmaceutical product of their own. On the other hand we have about 500 established pharma companies. Some 200 of them are interested in R\&D and innovation. These are mostly medium-sized companies. They have know-how in drug development, registration, production and marketing \& sales, and they focus on national and European markets. But because of the present health policy in Germany they are under strong pressure. The 'big' global pharma companies have left Germany within the last decade. There are less than ten left, but none of them is amongst the top ten players in the world.

To create value within the growing health care market, Germany has to concentrate on its strengths, remove its weaknesses and improve the environment for pharma R\&D.

\section{The 'Strategic Gap'}

The strengths in Germany are the excellent science and hundreds of biotechand pharma companies. But today's weakness is the 'strategic gap' between early development in science and start-up companies and the unwillingness of the estab-

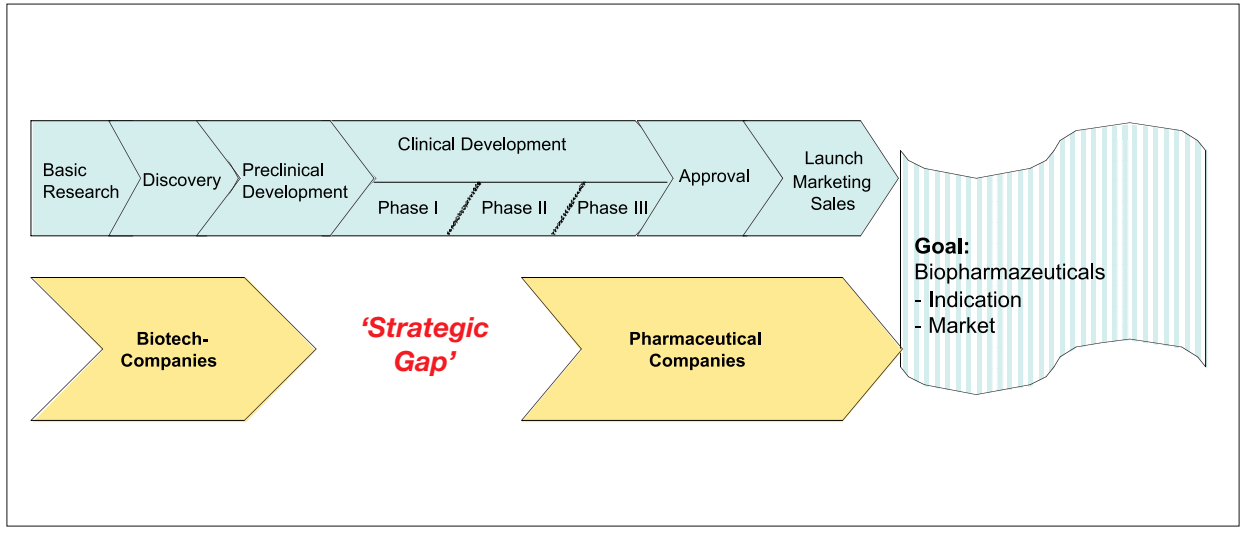

Fig. 1. The 'strategic gap' between discovery and clinical development

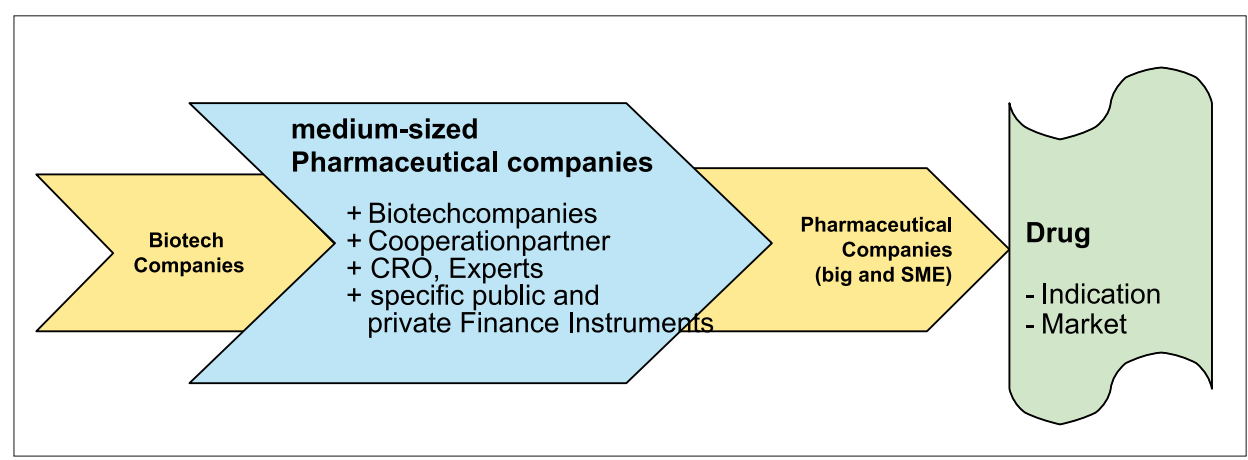

Fig. 2. Bridging the 'strategic gap' with 'competence modules'

lished pharmaceutical companies to take the risks of early development (Fig. 1).

As there are so many synergies between science, biotech and pharmaceutical companies, the best chance for innovation is cooperation. Challenge and opportunity in Germany is to establish an excellent technology transfer and to complete the value chain by joining 'competence modules' (Fig. 2).

Within this concept the medium-sized pharmaceutical companies can play an important roll and bridge together with further partners - the strategic gap.

\section{Creation of Value}

To top the full potential in Germany we have to ensure that basic discoveries turn into new and better medical treatments which we develop and produce in Germany and sell internationally. Therefore Germany has to implement an innovation-friendly framework, improve patenting and technology transfer, use synergies between biotech and pharma and increase innovation through cooperation and networking. This is the only way to create value and get back a little bit of the brilliance of the "pharmacy of the world' of former times.

Today there are some hurdles which have to be removed to bring the process forward. Most of the potential players do not know how to find the right partner and the adequate project, and where to get appropriate support. Communication between pharma and biotech and science has to be improved; they all think and live in different worlds. The politicians must improve the business environment for innovations, i.e. for the process of drug development and for reimbursement. However, the main hurdle is that there are not enough suitable finance instruments in the public and private field available.

\section{Support from the German Pharma Industry (BPI)}

The Association of the German Pharma Industry (Bundesverband der Pharmazeutischen Industrie, BPI) started three years ago with an initiative to support pharma- and biotech companies on the multidimensional path to innovation.

The BPI represents the full range of pharmaceutical companies in Germany: 
With its experience, networks, and the specific member database, the BPI helps to find the right partner and the adequate project. Additionally the BPI offers specific Pharma-biotech partnering forums. During these networking events the participants learn about pharma, biotech, and science in Germany. There is time for presentation and sharing of ideas and the chance to talk to each other! During the whole path from biotech ideas to commercial drugs including finance (public funds and private), patents, registration, safety, reimbursement and many more the BPI offers support to all member, supplemented by the BPI network of experts.

\section{Innovation through Cooperation}

To reach the end of stagnation in the field of pharmaceutical innovation, cooperation and networking between science, biotech, and pharma is essential: Science offers ideas for innovation, biotech companies offer expertise in drug discovery and pharma companies offer know-how in drug development, registration, production, marketing, sales, and reimbursement. To close the 'strategic gap' the availability of intelligent finance instruments is - beside pharma and biotech networking - corequisite. Without money many promising early developments and many cooperation opportunities between biotech and pharma are doomed to failure.

But the basic necessity is to enable financiers to make adequate venture capital available in Germany by the facilitation of the fiscal system for investors. If it is not attractive to invest in Germany, the investments are done in other parts of the world.

There are many innovative ideas for new financing models. During the session of the Business Platform BioValley 2004 in Freiburg experts discussed possibilities to manage the problems and outlined their ideas. Hopefully, some of them will be realized.

Received: October 22, 2004 